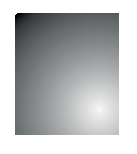

\title{
Determinantes e Probabilidades do Primeiro EMPREGO: UMA ABORDAGEM DA DEMANDA DAS empresas em Santa Catarina
}

Determining and Probability of the First Job: an approach to demand from companies in Santa Catarina

\author{
Magno Rogério Gomes \\ Mestre em Economia Regional na UEL/PR. Universidade Estadual de Londrina. Londrina, PR. Brasil. E-mail: magnorg86@gmail.com
}

\section{Solange de Cassia Inforzato de Souza}

Professora associada do Curso de Economia e do Programa de Mestrado em Economia Regional da Universidade Estadual de Londrina. Londrina, PR. Brasil.E-mail: solangecassia@uol.com.br

\section{Katy Maia}

Professora associada do Curso de Economia e do Programa de Mestrado em Economia Regional da Universidade Estadual de Londrina. Londrina, PR. Brasil. E-mail: katymaia@terra.com.br

\section{Resumo}

Este estudo tem por objetivo analisar a contratação dos trabalhadores no primeiro emprego pelas empresas formais privadas em Santa Catarina. Para isso, foram utilizados os microdados da RAIS 2012 - Relação Anual de Informações Sociais do Ministério do trabalho - $e$ aplica-se o modelo logit multinomial para a análise. Os resultados mostram que as empresas dos setores da indústria e agropecuária demandam, em geral, trabalhadores do sexo masculino, $e$ as do comércio e serviços, as mulheres. Essa demanda é também observada para os indivíduos jovens de 18 a 24 anos e com ensino médio concluído. As empresas do setor de serviços apresentam maior probabilidade de contratação de indivíduos com nível superior, de ambos os sexos, sendo mais intensa para os indivíduos não brancos. O incremento da escolaridade dos trabalhadores produz redução das diferenças de gênero na absorção na agricultura, comércio e indústria, mas aumenta no setor de serviços da economia catarinense.

Palavras-chave: Primeiro Emprego. Setores Econômicos. Santa Catarina.

\section{Abstract}

This study aims to examine the hiring of first job of workers by private formal companies in the state of Santa Catarina. For this, it uses the data from the RAIS 2012 - Annual Social Information Department of Labor and applies the multinomial logit model for the analysis. The results show that companies in the industry and agriculture sectors require, in general, male workers, and the sectors of trade and services, give priority to women. This demand is also observed for young people 18-24 years old with high school completed. The service sector companies are more probably to hiring individuals with higher education, for both sexes, being more intense for nonwhites. Increased education of workers produces reducing gender differences in absorption in agriculture, trade and industry, but increases in the services sectors of Santa Catarina economy.

Keywords: First Job. Economic Sectors. Santa Catarina. 


\section{INTRODUÇÃo}

A primeira inserção dos brasileiros no mercado de trabalho tem sido tema de estudos, seja pela utilização do potencial produtivo dessa população pelas empresas envolvidas, seja pela sua relevância como fator inibidor de práticas ilícitas diante das vulnerabilidades a que estão expostos os jovens na sociedade brasileira.

No entanto, segundo os dados da Pesquisa Nacional por Amostra de Domicílios 2012, a taxa de desemprego enfrentada pelos jovens de 15 a 17 anos de idade foi de $21 \%$ e para o jovem de 18 a 24 anos foi de $13,2 \%$, sendo $6,1 \%$ a média brasileira para todas as idades. Outras pesquisas mostram que muitos jovens não estão trabalhando ou procurando emprego, nem estudando no Brasil. O total de jovens entre 15 e 29 anos pertencentes à classe dos "nem-nem" é de 9,6 milhões de indivíduos, o que corresponde a 19,6\% da população brasileira dessa faixa etária, sendo a maioria mulheres, 70,3\% (IBGE, 2013). No estado de Santa Catarina, dos mais de 1,6 milhão de jovens na faixa etária de 15 a 29 anos, 9,8\% não estavam no mercado de trabalho e não estudavam em 2010, conforme Remy e Vaz (2014).

O trabalho na juventude ou na etapa mais avançada da idade da população pode estar relacionada à decisão de oferta de trabalho do indivíduo, mas também à demanda de trabalho das empresas nos diversos setores econômicos. A literatura empírica tem evidenciado que a escolha do trabalhador por parte do empregador leva em conta os fatores produtivos como a escolaridade, o treinamento e a experiência, como determinantes ou sinalizadores da produtividade de cada candidato ao emprego. Os perfis não produtivos também podem interferir, indicando possíveis discriminações de gênero e cor.

De acordo com a teoria do capital humano, a produtividade e os rendimentos de um trabalhador aumentam em função da elevação da escolaridade e de suas habilidades adquiridas com a experiência (BECKER, 1962). Por outro lado, para Cacciamali (1978), do ponto de vista da teoria da segmentação do mercado de trabalho, cada tipo de emprego ou empresa tem diferentes critérios de recrutamento $e$ as vagas são preenchidas por diferentes grupos de trabalhadores que constituem a força de trabalho. Doeringer e Piore
(1973), expoentes da teoria da segmentação, reforçam que são as características pessoais dos indivíduos como escolaridade, cor, gênero, experiência que determinam o tipo de mercado em que eles serão inseridos.

Além disso, a literatura aponta para outros fatores associados ao ciclo de vida e a origem socioeconômica do jovem, pois aqueles de famílias mais pobres são propensos a procurar um trabalho e abandonar os estudos. No lado da demanda, os segmentos do mercado de trabalho podem atribuir demandas mais ou menos estáveis, e diferentes níveis tecnológicos exigem qualificações e habilidades mais gerais ou mais específicas dos indivíduos.

Diante disso, o objetivo deste artigo é analisar a contratação dos trabalhadores no primeiro emprego pelas empresas formais privadas no estado de Santa Catarina, a partir das suas características pessoais. Para isso, utilizam-se os microdados da RAIS - Relação Anual de Informações Sociais do Ministério do trabalho de 2012 - e o modelo logit multinomial para análise das informações.

O trabalho está dividido em cinco seções, além desta introdução. Na segunda seção revisa-se a literatura sobre o assunto e na terceira apresenta-se a metodologia e base de dados. Na quarta seção descrevem-se as características dos primeiros empregos gerados no estado e discutem-se os resultados do modelo econométrico. Ao final, as conclusões são sumarizadas.

\section{Determinantes de uma Contratação: TEORIA E EVIDÊNCIAS}

No estudo da demanda por mão de obra, segundo a teoria econômica neoclássica, como o capital e a tecnologia de produção são fixos no curto prazo, uma decisão sobre o nível de emprego implica uma decisão sobre o nível de produção. A demanda por mão de obra é derivada da função produção das empresas $\mathrm{Y}=\mathrm{A} \mathrm{f}(\mathrm{K}, \mathrm{N})$, onde $(\mathrm{A})$ representa o fator tecnológico, (K) o capital/máquinas e equipamentos e $(\mathrm{N})$ mão de obra. Essa demanda é negativamente inclinada pelo fato de derivar da função de produção e, assim, quando o único fator de produção variável é a mão de obra, a demanda dos empregadores depende do valor dos salários e de sua produtividade. 
Segundo Borjas (2012) os trabalhadores divergem em suas características produtivas e não produtivas e os demandantes avaliam tais aspectos.

\begin{abstract}
Os trabalhadores são muito heterogêneos, pois alguns deles têm graus universitários, enquanto outros não terminaram o ensino médio; alguns tem muita experiência no mercado de trabalho, e outro são novatos. Em resumo, alguns trabalhadores provavelmente farão uma contribuição muito maior para a produção da empresa do que outros. (BORJAS, 2012, p. 97)
\end{abstract}

Para Doeringer e Piore (1973), expoentes da teoria da segmentação do mercado de trabalho, são as características pessoais dos indivíduos que determinam o tipo de mercado em que eles serão inseridos.

Empregos com características distintas são associados a diferentes pessoas. Características de indivíduos, como raça, sexo, background social, anos de escolarização, experiência no emprego, experiência na firma, etc., irão determinar na sua gama de oportunidade de trabalho. Sua alocação em um emprego, por sua vez, irá condicionar a evolução futura de suas características pessoais. (LIMA, 1980, p..237)

Outro enfoque dessa teoria, apresentado por Lima (1980), são os estudos de Barry Bluestone, de Bennet Harrison, e de Tomas Vietorisz, cuja preocupação repousa sobre o comportamento da estrutura industrial, dando mais ênfase às características dos postos de trabalho, das firmas e à interação entre os agentes. De acordo com Vietorisz e Harrison (1973), a segmentação surge pelas diferenças tecnológicas entre as atividades econômicas, pois existem atividades intensivas em tecnologia e qualificação de mão de obra e atividades intensivas em mão de obra de baixa qualificação.

A teoria dos mercados duais ou segmentados divide o mercado de trabalho em: mercado primário e secundário. O primário é caracterizado por grandes empresas oligopolistas, empregos estáveis, alta produtividade, intensivo em tecnologia, altos salários, em geral há planos de carreiras, investimentos em treinamento, trabalhadores sindicalizados e apresenta baixa rotatividade nesse mercado. O mercado secundário é definido por pequenas empresas competitivas com baixos lucros, baixos salários, baixa produtividade, intensivo em mão de obra, alta rotatividade, estagnação tecnológica, má condições de trabalho, não existe plano de carreira, não há investimento em treinamentos, trabalhadores não sindicalizados etc.

Empiricamente, vários estudos mostram as características produtivas e não produtivas que os empregadores levam em consideração na ocasião de uma contratação, pois

Cada tipo de emprego caracteriza-se por diferentes critérios de recrutamento, seleção, treinamento e promoção de mão de obra, bem como apresenta diversas formas de supervisão, condições de trabalho e níveis salariais. São por outro lado, preenchidos por diferentes grupos de trabalhadores que constituem a força de trabalho. (CACCIAMALI, 1978, p. 59)

Casari (2012) trata de estudo realizado sobre a segmentação ocupacional na agricultura das Filipinas para os anos de 1984-85 que conclui que das características observadas dos trabalhadores provêm informações sobre sua produtividade quando não há informação completa para os empregadores. O autor expõe outra pesquisa em que as ocupações são determinadas em termos de habilidades e aptidões, mas que, normalmente, a alocação dos trabalhadores tem caráter social e cultural, levando à diferenciação por gênero. As empresas podem atribuir demandas mais ou menos estáveis e diferentes níveis tecnológicos exigem qualificações e habilidades mais gerais ou mais especificas dos indivíduos.

No Brasil, Cacciamali (1978), Kon (2004) e Kon, Leite e Marques (2011), identificam as características que irão determinar as escolhas por parte do empregador, como a escolaridade do candidato, experiência, idade, gênero, raça, status sócio econômico, entre outras. Homens de maior escolaridade, experiência profissional e status sócio econômico obterão os melhores empregos, e os indivíduos homens e mulheres, entre os menos favorecidos da sociedade, desempenharão empregos secundários.

Portanto, seguindo a teoria da segmentação, é de ser esperar que os ingressantes no mercado de trabalho, particularmente os jovens, tendam a ser alocados nos empregos secundários, que normalmente são fornecidos por pequenas empresas competitivas, intensivas em mão de obra, com baixos salários, e sem planos de carreira. A situação é pior para jovens de condição 
socioeconômica desfavorecida, pois de acordo com Remy e Vaz (2014) e Pochmann (2003), jovens de familias pobres tendem a abandonar os estudos e entrar no mercado de trabalho precocemente.

Segundo Casari (2012), o maior volume de recrutamento de novos trabalhadores é para cargos em que o nível de habilidades exigido é baixo e, para os cargos demandantes de maiores habilidades, são promovidos funcionários que foram treinados ou que obtiveram o conhecimento necessário através de sua experiência anterior dentro do mercado de trabalho interno. Além disso, DIEESE (2009) e Carvalho (2004) constataram que as mulheres e os negros são valorados negativamente no mercado de trabalho, ao compará-los com os homens e os brancos. A inserção ocupacional dos dois grupos citados está em grande proporção atribuída a cargos poucos qualificados e a salários baixos.

Bousquat e Cohn (2003) mostraram que em São Paulo no ano de 2003, $16 \%$ dos jovens das regiões pobres trabalhavam na indústria, enquanto para os jovens ricos esse setor absorvia $4,4 \%$. Já no setor de Serviços, $45 \%$ eram jovens de classes mais pobres e $51,5 \%$ eram jovens de classes mais ricas. Na ocupação agrícola, no Brasil, entre 2004 e 2009, pessoas mais velhas, homem, da cor parda e pouca escolaridade aumentam a probabilidade do indivíduo ter uma ocupação; se negro esta chance se reduz, segundo Casari (2012).

Em suma, a literatura documenta uma dualidade existente no mercado de trabalho entre empregos primários e secundários, e segmentação dos postos de trabalho por setores econômicos. Mulheres e trabalhadores jovens e sem experiência seriam mais demandados em segmentos secundários e setores econômicos menos favorecidos em termos salariais.

\section{Metodologia e Base de Dados}

Para esta investigação foram utilizados os microdados da RAIS - Relação Anual de Informações Sociais do Ministério do trabalho de 2012, para o estado de Santa Catarina (SC). A RAIS é realizada por pesquisa de estabelecimentos no Brasil e traz informações sobre o mercado de trabalho formal brasileiro, utilizada na elaboração de políticas públicas relacionadas ao emprego e renda.
Dessa base de dados foram extraídos apenas os indivíduos com mais de 15 e menores de 65 anos que foram contratados no ano e mantinham vínculo empregatício em 31 de dezembro de 2012, excluídos os indivíduos pertencentes à administação pública $e$ domésticas. No que se refere ao porte das empresas, seguiu-se o critério do SEBRAE (2013) por número de empregados: para o setor da Indústria, classifica-se como Microempresa (até 19 ocupados); Pequena empresa (de 20 a 99 ocupados); Média empresa (de 100 a 499 ocupados); Grande empresa (500 ou mais ocupados). Para o setor de Serviços e Comércio segue a seguinte classificação: Microempresa (até 9 ocupados); Pequena empresa (de 10 a 49 ocupados); Média empresa (de 50 a 99 ocupados); Grande empresa (100 ou mais ocupados).

A análise contempla quatro setores econômicos no âmbito privado: em setor da Indústria (Construção Civil; Extrativo Mineral e Indústria de Transformação; Serviços Industriais de Utilidade Pública), setor do Comércio (Comércio), setor de Serviços (Serviços) e, por fim, o setor Agrícola (Agropecuária, Extrativo Vegetal, Caça e Pesca).

A análise empírica foi realizada pelo método the multinominal logit model, o modelo logístico multinominal, que nos fornece a probabilidade de um indivíduo estar empregado em um determinado setor em relação a outro setor, de acordo com suas características.

O modelo multinomial tem sido muito utilizado em pesquisas brasileiras, o que reforça a sua escolha neste trabalho. Exemplos de seu uso podem ser encontrados em Fernandes e Pichetti (1999), Oliveira, Scorzafave e Pazello (2009), Casari (2012), Silva e Kassouf (2002), Tomás (2007) e Mendonça et al. (2012).

\subsection{Modelo Logit Multinomial}

De acordo com Long e Freese (2001), Barreiro, Ruzo e Losada (2004) e Fávaro et al. (2014), o modelo de regressão logística consiste em que a variável dependente deva ter mais de duas categorias e a resposta tanto pode ser nominal quanto ordinal. As variáveis explicativas podem ser categóricas ou quantitativas. Em modelos multinomiais presume-se que as categorias (variáveis dependentes) tenham uma distribuição multinomial, o que, por sua vez, é uma generalização de uma distribuição binomial. 
Formalmente, o modelo logit multinomial em Greene (2012) supõe que há $k$ categorias para variável independente, e a categoria 1 é tida como base; as probabilidades são dadas por:

$$
p_{i j}=\operatorname{Pr}\left(y_{j}=i\right)=\left\{\begin{array}{l}
\frac{1}{1+\sum_{m=2}^{k} e^{\left(x_{j} \beta_{m}\right)}}, \text { se } i=1 \\
\frac{e^{\left(x_{j} \beta_{i}\right)}}{1+\sum_{m=2}^{k} e^{\left(x_{j} \beta_{m}\right)}}, \text { se } i>1
\end{array}\right.
$$

Em que,

i é o número de equações que são resolvidas para determinar a probabilidade; $x_{j}$ é um vetor linha que compreende o conjunto de variáveis explicativas associada à observação j; $e \beta_{m}$ é o vetor de coeficientes das categorias.

Os efeitos marginais podem ser expressos por:

$$
\frac{\partial\left(\operatorname{Pr}\left(y_{j}=i \mid X\right)\right)}{\partial x_{j}}=\operatorname{Pr}\left[y_{j}=i \mid X\right]\left(\beta_{i} \sum_{m=2}^{k} \beta_{k} \operatorname{Pr}\left[y_{j}=k \mid X\right]\right)
$$

Para este estudo, o setor definido como base foi o setor de Serviços, na comparação com os demais.

Uma abordagem alternativa de análise é a chamada odds-ratio ou razão de risco relativo (RRR). Define-se a (RRR) como a probabilidade de dado evento ocorrer, em relação a outro. Assim, considera-se a probabilidade de ocorrência do evento e a probabilidade de não ocorrência. Portanto, a razão de chance no modelo logit multinomial, segundo Mendonça et al. (2012) é definida por:

$$
R R R=\frac{\frac{\operatorname{pr}(Y=j / x+1)}{\operatorname{pr}(Y=k / x+1)}}{\frac{\operatorname{pr}(Y=j / x)}{\operatorname{pr}(Y=k / x)}}
$$

Para facilitar a interpretação, a odds ratio pode ser convertida em incremento percentual, o que vem a fornecer a probabilidade de mudança da categoria base para a categoria analisada em função das variações nas características dos indivíduos, da seguinte forma, segundo Mendonça et al. (2012):

$$
(O d d s-1) * 100
$$

\begin{tabular}{|c|c|}
\hline $\begin{array}{c}\text { DEPENDENTE - } \\
\text { CATEGORIAS }\end{array}$ & EXPLICATIVAS \\
\hline Agricultura & $\begin{array}{c}\text { Raça/Cor }-(\text { Branco }=1 ; \text { Não branco }=0) \\
- \text { Excluídos indígenas e ignorados }\end{array}$ \\
\hline Comércio & Gênero $-($ Homem $=1 ;$ Mulher $=0)$ \\
\hline Serviços & $\begin{array}{l}\text { Experiência - (Idade - Anos de } \\
\text { estudo - 5). Para indivíduos que foram } \\
\text { contratados no primeiro emprego, } \\
\text { foi atribuído }(0) \text { à experiência }\end{array}$ \\
\hline \multirow[t]{12}{*}{ Indústria } & Experiência ${ }^{2}$ \\
\hline & Analfabeto - (menos de um ano de estudo) \\
\hline & $\begin{array}{l}\text { Primário - (igual ou menos } \\
\text { que } 5 \text { anos de estudo) }\end{array}$ \\
\hline & $\begin{array}{l}\text { Ensino fundamental - (igual ou } \\
\text { menos que } 9 \text { anos de estudo) }\end{array}$ \\
\hline & Ensino Médio - (Variável omitida) \\
\hline & $\begin{array}{l}\text { Ensino Superior - (Graduação, } \\
\text { Mestrado e Doutorado) }\end{array}$ \\
\hline & Idade 15 a 17 \\
\hline & Idade 18 a 24 - (Variável omitida) \\
\hline & Idade 25 a 29 \\
\hline & Idade 30 a 39 \\
\hline & Idade 40 a 49 \\
\hline & Idade 50 a 64 \\
\hline
\end{tabular}

\subsection{Descrição das Variáveis do Modelo}

\section{Resultados e Discussão}

Nesta seção realiza-se a caracterização dos empregos gerados no estado de Santa Catarina e a mensuração das probabilidades de contratação no primeiro emprego

\subsection{Características dos Empregos Gerados em Santa Catarina}

As informações sobre os empregos gerados no ano de 2012 nos dão conta de que a região sul do Brasil contribuiu com mais de $18 \%$ do primeiro emprego e reemprego, do total de aproximadamente 14 milhões gerados no Brasil. As primeiras oportunidades de emprego corresponderam a $13,67 \%$ das contratações no ano (2.529.370). 
Em uma análise por setores e por gênero para o primeiro emprego na região sul (Tabela 1), pode-se afirmar que os homens tiveram maior oportunidade ao primeiro emprego, pois dos 346 mil postos de trabalho, mais de $54 \%$ foram preenchidos por indivíduos do sexo masculino, e aproximadamente $46 \%$ pelo sexo feminino. O maior ingresso feminino no primeiro emprego foi no setor de Serviços, com mais de $53 \%$ em comparação com o sexo masculino.

Tabela 1 - Primeiro emprego por gênero nos setores econômicos, na região sul no ano de 2012

\begin{tabular}{|c|c|c|c|c|c|c|}
$\begin{array}{c}\text { Setores } \\
\text { Econômicos }\end{array}$ & \multicolumn{5}{|c|}{ GÊnero } \\
\cline { 2 - 8 } & Mulher & $\mathbf{\%}$ & Homem & $\mathbf{\%}$ & TotAl & $\%$ \\
\hline Agrícola & 3.602 & $\mathbf{2 8 , 8 5}$ & 8.885 & $\mathbf{7 1 , 1 5}$ & 12.487 & $\mathbf{1 0 0}$ \\
\hline Comércio & 54.851 & $\mathbf{4 8 , 9 4}$ & 57.225 & $\mathbf{5 1 , 0 6}$ & 112.076 & $\mathbf{1 0 0}$ \\
\hline Serviços & 60.517 & $\mathbf{5 3 , 3 9}$ & 52.828 & $\mathbf{4 6 , 6 1}$ & 113.345 & $\mathbf{1 0 0}$ \\
\hline Indústria & 39.690 & $\mathbf{3 6 , 7 6}$ & 68.278 & $\mathbf{6 3 , 2 4}$ & 107.968 & $\mathbf{1 0 0}$ \\
\hline Total & $\mathbf{1 5 8 . 6 6 0}$ & $\mathbf{4 5 , 8 7}$ & $\mathbf{1 8 7 . 2 1 6}$ & $\mathbf{5 4 , 1 3}$ & $\mathbf{3 4 5 . 8 7 6}$ & $\mathbf{1 0 0}$ \\
\hline
\end{tabular}

Fonte: Elaborada pelos autores deste artigo, a partir dos dados da RAIS/MTE, 2012

A grande divergência de contratação de mão de obra sem experiência, por gênero, está no setor Agrícola, no qual $71,15 \%$ das vagas foram preenchidas por homens e $28,85 \%$ por mulheres. Em outras palavras, para cada 2,46 homens empregados, o setor empregava apenas uma mulher. Outro setor que aparentemente apresentou uma característica de preferência pelo sexo masculino foi o da Indústria, pois, dos postos de trabalho gerados, $63,24 \%$ ficaram com os homens e $36,76 \%$ com as mulheres, uma diferença percentual de mais de 26 pontos percentuais em favor dos homens.

No Estado de Santa Catarina, dos mais de $90 \mathrm{mil}$ novos primeiros empregos gerados, o setor industrial empregou $34,97 \%$, seguido do setor dos Serviços $(31,93 \%)$ e Comércio (30,37\%). No que tange à ocupação por gênero, os setores Agrícola, Comércio e Indústria apresentaram um resultado de divergência na alocação da mão de obra sem experiência em favor dos homens para esses três setores; o setor de Serviços é que priorizou a contratação das mulheres (Tabela 2).

A maior diferença percentual está no setor Agrícola, em que das 2,4 mil primeiras oportunidades geradas no setor, $63,85 \%$ foram preenchidas por homens e $36,15 \%$ por mulheres. Outro setor que apresentou uma diferença percentual significativa foi a Indústria que priorizou o sexo masculino com $60,13 \%$ das mais de 31,5 mil oportunidades de primeiro emprego; a mulher ficou com $39,87 \%$ deste total de empregos gerados.

Tabela 2 - Primeiro emprego por gêneros nos setores econômicos no estado de SC, em 2012

\begin{tabular}{|c|c|c|c|c|c|c|}
\hline \multirow{2}{*}{$\begin{array}{c}\text { Setores } \\
\text { Econômicos }\end{array}$} & \multicolumn{2}{|c|}{ MulheR } & \multicolumn{2}{|c|}{ НОМЕм } & \multicolumn{2}{|c|}{ TOTAL } \\
\hline & $\begin{array}{c}\text { ConTRA- } \\
\text { TADOS }\end{array}$ & $\%$ & $\begin{array}{c}\text { CONTRA- } \\
\text { TADOS }\end{array}$ & $\%$ & $\begin{array}{c}\text { CONTRA- } \\
\text { TADOS }\end{array}$ & $\%$ \\
\hline Agrícola & 891 & 36,15 & 1.574 & 63,85 & 2.465 & 2,73 \\
\hline Comércio & 13.408 & 48,89 & 14.018 & 51,11 & 27.426 & 30,37 \\
\hline Serviços & 15.357 & 53,25 & 13.485 & 46,75 & 28.842 & 31,93 \\
\hline Indústria & 12.591 & 39,87 & 18.990 & 60,13 & 31.581 & 34,97 \\
\hline & & & & & 90.314 & \\
\hline
\end{tabular}

Fonte: Elaborada pelos autores deste artigo, a partir dos dados da RAIS/MTE, 2012

Na região Sul, indivíduos contratados pelo primeiro emprego com ensino médio foram os que mais ingressaram no mercado de trabalho, $67,38 \%$, seguido pelos de ensino fundamental, com $17,59 \%$. As pessoas com graduação, que conseguiram o primeiro emprego foi $10,62 \%$ do total da região.

As empresas que mais empregaram trabalhadores com ensino fundamental no estado de Santa Catarina foram as que estão reunidas na Indústria, Serviços e Comércio (Gráfico 1). Para a faixa de escolaridade de ensino médio, Santa Catarina apresentou o maior percentual relativo de trabalhadores empregados no Comércio, seguido pela Indústria e Serviços.

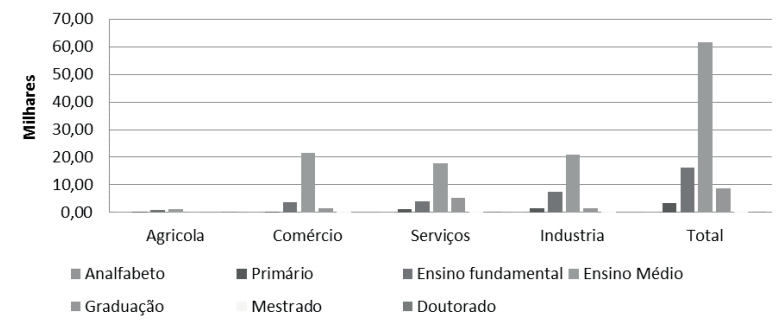

Gráfico 1- Primeiro emprego por nível de escolaridade e setores econômicos, em SC, 2012

Fonte: Elaborado pelos autores deste artigo, a partir dos dados da RAIS/MTE, 2012

No que se refere à idade dos trabalhadores que foram contratados pela primeira vez no estado de 
Santa Catarina, no ano de 2012, os resultados estão apresentados na Tabela 3.

Tanto no estado de SC e como na região Sul os indivíduos que pertencem à faixa etária de 18 a 24 anos foram os priorizados na oportunidade do primeiro emprego (pouco mais de $42 \%$ para a região e $41,05 \%$ para SC). Outra faixa etária que apresentou mais de $21 \%$ dos contratados na região foram indivíduos que tinham idade ente 15 a 17 anos

Tabela 3 - Faixa etária dos contratados na Região Sul do Brasil e em Santa Catarina, 2012

\begin{tabular}{|c|c|c|c|c|}
\hline Faixa EtáRIA & $\mathbf{S C}$ & $\mathbf{\%}$ & Total - Região & $\mathbf{\%}$ \\
\hline 15 a 17 anos & 22412 & $\mathbf{2 4 , 8 2}$ & 74579 & $\mathbf{2 1 , 5 6}$ \\
\hline 18 a 24 anos & 37071 & $\mathbf{4 1 , 0 5}$ & 146295 & $\mathbf{4 2 , 3}$ \\
\hline 25 a 29 anos & 10051 & $\mathbf{1 1 , 1 3}$ & 40208 & $\mathbf{1 1 , 6 2}$ \\
\hline 30 a 39 anos & 11987 & $\mathbf{1 3 , 2 7}$ & 48622 & $\mathbf{1 4 , 0 6}$ \\
\hline 40 a 49 anos & 6089 & $\mathbf{6 , 7 4}$ & 24738 & $\mathbf{7 , 1 5}$ \\
\hline 50 a 64 anos & 2704 & $\mathbf{2 , 9 9}$ & 11434 & $\mathbf{3 , 3 1}$ \\
\hline Total & 90314 & $\mathbf{1 0 0}$ & 345876 & $\mathbf{1 0 0}$ \\
\hline$\%$ & & $\mathbf{2 6 , 1 1}$ & & $\mathbf{1 0 0}$ \\
\hline
\end{tabular}

Fonte: Elaborada pelos autores deste artigo, a partir dos dados da RAIS/MTE, 2012

Os dados confirmam que $77 \%$ dos indivíduos contratados no primeiro emprego em Santa Catarina são jovens entre 15 e 29 anos, e os ingressantes na faixa de 15 a 24 anos de idade representaram $66 \%$ dos contratados. Um fator determinante para isso pode ser os programas governamentais que visam dar a primeira oportunidade para os jovens como: o Programa Nacional do Primeiro Emprego (PNPE) e também a "Lei do Aprendiz", Lei Federal 10.097/2000; que determina que empresas de médio e grande porte são obrigadas a contratar numa proporção de $5 \%$ a $15 \%$ jovens aprendizes no seu quadro de colaboradores.

Os resultados da absorção dos trabalhadores por porte das empresas (Tabela 4), seguindo o critério do SEBRAE (2013), são os seguintes: as Micro e Pequenas Empresas da Indústria, Comércio e Serviços, na região Sul, contrataram $48,18 \%$ dos novos trabalhadores, $e$ em Santa Catarina 48\%. Portanto, adultos e, em sua maioria, os jovens sem experiência que buscam uma oportunidade são requeridos pelo mercado secundário, corroborando a teoria da segmentação, onde os empregos são gerados por pequenas empresas $e$ baixos salários.

Tabela 4 - Absorção do primeiro emprego por porte de empresas, na Região Sul e em Santa Catarina, 2012

\begin{tabular}{|c|c|c|c|c|}
\hline Porte DAS EMPREGAS & SC & $\mathbf{\%}$ & $\begin{array}{c}\text { Total - } \\
\text { ReGião }\end{array}$ & $\%$ \\
\hline Micro - Indústria & 6034 & $\mathbf{6 , 6 8}$ & 20598 & $\mathbf{5 , 9 6}$ \\
\hline Pequena - Indústria & 8828 & $\mathbf{9 , 7 7}$ & 29832 & $\mathbf{8 , 6 3}$ \\
\hline Média - Indústria & 6984 & $\mathbf{7 , 7 3}$ & 25003 & $\mathbf{7 , 2 3}$ \\
\hline Grande - Indústria & 523 & $\mathbf{5 , 7 9}$ & 17824 & $\mathbf{5 , 1 5}$ \\
\hline Micro - Comercio/Serviços & 11948 & $\mathbf{1 3 , 2 3}$ & 48327 & $\mathbf{1 3 , 9 7}$ \\
\hline Pequena - Comercio/ & 16527 & $\mathbf{1 8 , 3}$ & 67868 & $\mathbf{1 9 , 6 2}$ \\
\hline Serviços & 7868 & $\mathbf{8 , 7 1}$ & 33419 & $\mathbf{9 , 6 6}$ \\
\hline Média - Comercio/Serviços & 1646 & $\mathbf{1 8 , 2 3}$ & 63537 & $\mathbf{1 8 , 3 7}$ \\
\hline Grande - Comercio/Serviços & 10435 & $\mathbf{1 1 , 5 5}$ & 39468 & $\mathbf{1 1 , 4 1}$ \\
\hline Outras & 90314 & $\mathbf{1 0 0}$ & 345876 & $\mathbf{1 0 0}$ \\
\hline Total & & & & \\
\hline
\end{tabular}

Fonte: Elaborada pelos autores deste artigo, a partir dos dados da RAIS/MTE, 2012

A média de salários pagos, em 2012, aos ingressantes no primeiro emprego na região sul foi de $R \$ 988,33$, sendo que o setor de Serviços pagou $R \$$ 1163,00, seguido do setor da Indústria, $\mathrm{R} \$ 961,69$, Agrícola, $\mathrm{R} \$ 891,90$, e o setor do Comércio, $\mathrm{R} \$ 848,08$. No Estado de Santa Catarina, o salário médio pago aos iniciantes no mercado de trabalho foi de $\mathrm{R} \$ 1.000,22$. O setor de Serviços é o que melhor remunera os indivíduos que engajaram em seu primeiro emprego, com uma média salarial para o setor de $\mathrm{R} \$ 1.133,33$.

As mulheres admitidas em seu primeiro emprego ganhavam menos que os homens, e os indivíduos de cor preta e parda do mesmo gênero receberam em média menos que os indivíduos amarelos e brancos (Tabela 5). A pior situação foi da mulher preta que recebeu a mais baixa remuneração; o homem preto foi o que menos recebeu na comparação com os demais de seu gênero, entretanto, sua remuneração ainda é maior que a da mulher branca, o que sinaliza maior discriminação salarial no primeiro emprego entre os gêneros do que entre a raça/cor. 
Tabela 5 - Média salarial dos trabalhadores no primeiro emprego, por cor e gênero, na região Sul, em 2012

\begin{tabular}{|c|c|c|}
\hline CoR & MuLHER - (R\$) & HoMEM - (R\$) \\
\hline Amarela & 968,63 & $1.061,95$ \\
\hline Branca & 880,14 & $1.020,58$ \\
\hline Parda & 794,07 & 945,54 \\
\hline Preta & 771,58 & 903,65 \\
\hline
\end{tabular}

Fonte: Elaborado pelos autores deste artigo, a partir dos dados da RAIS/MTE, 2012

Os rendimentos médios dos trabalhadores em seus primeiros empregos formais gerados em Santa Catarina, no ano de 2012, por setor e gênero, estão descritos na Tabela 6. O setor que melhor remunerou mulheres e homens que ingressaram no mercado de trabalho foi o setor de Serviços, embora o salário médio das mulheres seja menor e a diferença entre os sexos, maior. O setor Agrícola foi o que apresentou a menor remuneração média para as mulheres e homens.

Tabela 6 - Rendimentos médios por setor e gênero, em Santa Catarina, 2012

\begin{tabular}{|c|c|c|c|}
\hline Setores & MulHeR - (R\$) & Homem - (R\$) & DiferENÇA - \% \\
\hline Agricultura & 772,93 & 888,82 & 14,99 \\
\hline Comércio & 866,67 & 903,94 & 4,30 \\
\hline Serviços & $1.030,23$ & $1.250,75$ & 21,40 \\
\hline Indústria & 883,79 & $1.060,54$ & 20,00 \\
\hline
\end{tabular}

Fonte: Elaborada pelos autores deste artigo, a partir dos dados da RAIS/MTE, 2012.

Em resumo, pode-se dizer que mais de $77 \%$ dos contratados para o primeiro emprego em Santa Catarina são jovens de 15 a 29 anos; a maior demanda de trabalhadores homens é na indústria, e de mulheres nos serviços; quase $50 \%$ dos trabalhadores foram inseridos em micro e pequenas empresas. Na indústria prevalece a ocupação dos trabalhadores com ensino fundamental e nos serviços, os graduados; o setor de serviços é o que melhor remunera homens e mulheres, mas apresenta a maior diferença de salários médios em favor dos homens, $21,4 \%$. O setor que aparentemente menos diferenciou os salários por gênero foi o setor do Comércio, 4,3\% em favor dos homens; e, por fim, há maior indicação de discriminação salarial por gênero do que por cor no estado.

\subsection{Possibilidades de contratação dos trabalhadores para o primeiro emprego: os efeitos marginais}

A partir da demanda realizada pelas empresas no estado de Santa Catarina em 2012, é possível estabelecer as chances da contratação dos trabalhadores no primeiro emprego pelas empresas nos setores econômicos, comparadas às do setor de serviços, cujos resultados da regressão logit multinomial constam no apêndice A1.

As probabilidades de um indivíduo ser contratado, a partir de suas características pessoais (efeitos marginais), estão apresentados na Tabela 7. No geral, um indivíduo sem experiência em Santa Catarina tem $1,9 \%$ de probabilidade de fazer parte do setor Agrícola, $25,2 \%$ de estar no setor do Comércio, e 34,28\% de pertencer ao setor de Serviços. Para o setor da Indústria a probabilidade de um indivíduo fazer parte desse setor foi de 38,61\% no estado de Santa Catarina em 2012.

Do ponto de vista das características dos trabalhadores, ser de cor preta reduz as chances de ser contratado no setor Agrícola, Comércio e Indústria em $0,5 \%, 6,0 \%$ e $1,5 \%$, respectivamente. Para o setor de Serviços, o efeito é positivo em $8,0 \%$. Ser da cor parda reduz a chance de ser contratado apenas no setor do Comércio, $4,6 \%$.

Os resultados também evidenciam que se os indivíduos forem do sexo masculino, reduz-se a probabilidade desses indivíduos pertencerem aos setores de Comércio e de Serviços em 4,6\% e 6,6\% respectivamente. Em relação aos setores Agrícola e da Indústria, se o trabalhador for do sexo masculino contribuirá para aumentar a probabilidade de fazer parte desses setores em $1,0 \%$ e $10,5 \%$, respectivamente.

Com relação às faixas de escolaridade (ensino médio - variável omitida), os resultados mostram que a probabilidade de pertencer à categoria Agrícola tornou-se maior se o indivíduo for analfabeto $(10,35 \%)$, se tiver o ensino primário $(9,88 \%)$ ou fundamental (3,36\%); e torna-se menor se tiver o nível superior (-1,5\%). Já para o setor da Indústria, a probabilidade de pertencer a este setor tornou-se maior se o indivíduo for analfabeto $(14,96 \%)$, se tiver primário $(10,54 \%)$ ou fundamental $(12,17 \%)$, e reduz para o indivíduo que tenha nível superior $(14,36 \%)$. 
Para o setor de Comércio, ser analfabeto $(15,76 \%)$, ter cursado o primário (17,33\%), fundamental $(10,0 \%)$ ou superior $(9,04 \%)$, reduz a probabilidade de fazer parte deste setor, comparado com o ensino médio. O setor de Serviços segue: ser analfabeto $(9,54 \%)$, primário $(3,08 \%)$ ou fundamental $(5,52 \%)$ reduz a probabilidade de pertencer ao setor analisado, e o indivíduo que tenha nível superior de escolaridade é mais propenso a fazer parte do setor de Serviços, em $24,9 \%$.

Tabela 7 - Efeitos Marginais para as Equações dos setores, Agrícola, Comércio, Serviços, Indústria - Santa Catarina, 2012

\begin{tabular}{|c|c|c|c|c|}
\hline \multirow{2}{*}{ VARIÁVEIS } & \multicolumn{4}{|c|}{ Setores - Santa Catarina } \\
\hline & Agrícola & Сomércio & Serviços & INDÚSTRIA \\
\hline Preta & $-0,0051^{*}$ & $-0,0605^{*}$ & $0,0805^{*}$ & $-0,0149 *$ \\
\hline Parda & $0,0069 *$ & $-0,0463^{*}$ & $0,0411^{*}$ & $-0,0018^{\mathrm{ns}}$ \\
\hline Homem & $0,0099 *$ & $-0,0495^{*}$ & $-0,0657^{*}$ & $0,1053^{*}$ \\
\hline Experiência & $-0,0003^{\text {ns }}$ & $-0,0003^{\text {ns }}$ & $0,0008 *$ & $-0,0001^{\mathrm{ns}}$ \\
\hline Experiência2 & $0,0000 *$ & $0,0000^{\mathrm{ns}}$ & $0,0000^{*}$ & $0,0000^{*}$ \\
\hline Analfabeto & $0,1035^{*}$ & $-0,1576^{*}$ & $-0,0954^{*}$ & $0,1496^{*}$ \\
\hline Primário & $0,0988^{*}$ & $-0,1733^{*}$ & $-0,0308^{*}$ & $0,1054^{*}$ \\
\hline Fundamental & $0,0336^{*}$ & $-0,1000^{*}$ & $-0,0552^{*}$ & $0,1217^{*}$ \\
\hline Superior & $-0,0150 *$ & $-0,0904 *$ & $0,2490 *$ & $-0,1436^{*}$ \\
\hline Idade15_A_17 & $0,0013^{\mathrm{ns}}$ & $0,1044 *$ & $-0,0344^{*}$ & $-0,0713^{*}$ \\
\hline Idade25_A_29 & $0,0024^{*}$ & $-0,0298^{*}$ & $0,0517^{*}$ & $-0,0244 *$ \\
\hline Idade30_A_39 & $0,0060 *$ & $-0,0465^{*}$ & $0,0712^{*}$ & $-0,0307 *$ \\
\hline Idade40_A_49 & $0,0101^{*}$ & $-0,0537^{*}$ & $0,0747^{*}$ & $-0,0311^{*}$ \\
\hline Idade50_A_64 & $0,0119 *$ & $-0,0497^{*}$ & $0,0977^{*}$ & $-0,0599 *$ \\
\hline Média & 0,0188* & $0,2522 *$ & $0,3428 *$ & $\mathbf{0 , 3 8 6 1 *}$ \\
\hline
\end{tabular}

Nota2: *significância $5 \%$; ns: não significativo

Fonte: Tabela elaborada pelos autores deste artigo a partir dos resultados da pesquisa

No que se refere às faixas de idade, omitida a dummy (18 a 24 anos), nota-se que pertencer a uma faixa de idade superior à omitida, colabora para que os indivíduos façam parte do setor Agrícola, e o mesmo ocorre em relação ao setor de Serviços. Para o setor de Comércio, a característica é oposta, isto é, fazer parte de uma faixa etária superior a 18 e 24 anos de idade reduz a chance de pertencer ao setor de Comércio; já para a faixa inferior, o sinal é positivo indicando um aumento da probabilidade. Por fim, para o setor da Indústria todas as faixas etárias apresentaram sinais negativos, o que evidencia que indivíduos que não estejam entre 18 a 24 anos de idade tem menos probabilidade de fazer parte deste setor.

Em síntese, as probabilidades de contratação pelas empresas do setor de serviços são maiores para os negros e pardos, as mulheres, os mais escolarizados e com mais de 24 anos de idade. No setor industrial, as demandas são para homens, brancos, baixa escolaridade e idade entre 18 e 24 anos. No comércio, as chances são maiores para brancos, mulheres, com ensino médio e com idade abaixo da faixa de 18 e 24 anos. Finalmente, na agricultura, a demanda maior é por brancos e pardos, homens, com pouca escolaridade e com idade superior à faixa de 18 a 24 anos.

Essas informações sinalizam a importância das características do trabalhador, como destacado pelos estudiosos referenciados neste artigo, na alocação dos postos de trabalho e, mais especificamente, tratados nesta pesquisa, nas empresas e seus setores econômicos em Santa Catarina.

O próximo passo foi analisar as probabilidades pontuais de contratação de pessoas sem experiência e em postos formais privados em Santa Catarina, nos setores econômicos, a partir das seguintes características dos indivíduos: (i) faixas etárias de 18 a 24 anos e 25 a 29 anos, com ensino médio, sexo e cor de pele; (ii) faixas etárias de 18 a 24 anos e 25 a 29 anos, com ensino superior, sexo e cor de pele. As figuras que seguem levam conta o (HB) homem branco, (HP) homem pardo, (HN) homem negro, (MB) mulher branca, (MP) mulher parda, (MN) mulher negra e (Dif. H-M) diferença entre homem e mulher.

Para indivíduos com idade de 18 a 24 anos e com ensino médio (Figura 1a), as considerações a fazer são as de que: homens e brancos tem maior probabilidade de encontrar o primeiro emprego na Indústria, $42,94 \%$. Para homens pardos esse percentual aumenta, $43,7 \%$, já para homens negros o percentual reduz, $42,85 \%$. Para as mulheres brancas, a maior probabilidade de encontrar o primeiro emprego está no setor do Comércio $(37,24 \%)$, e para as não brancas com as mesmas características, a maior probabilidade é de ingressar no setor de Serviços. 
Ao analisar as diferenças restritas ao sexo, os setores de Comércio e Serviços mostram-se mais propensos a admitir trabalhadores do sexo feminino, $e$ a Indústria mais trabalhadores do sexo masculino. No setor de Serviços, Santa Catarina prioriza em 5,04\% em favor do sexo feminino; no Comércio, a probabilidade de contratar mulher é de 6,33\% a mais do que homens.
Para o setor da Indústria, a preferência é por indivíduos do sexo masculino (probabilidade de $10,64 \%$ em comparação com o sexo feminino). O setor da Agricultura foi o mais homogêneo, tanto para a região como para o estado, apresentando menor diferença na absorção de mão de obra de homens e mulheres.

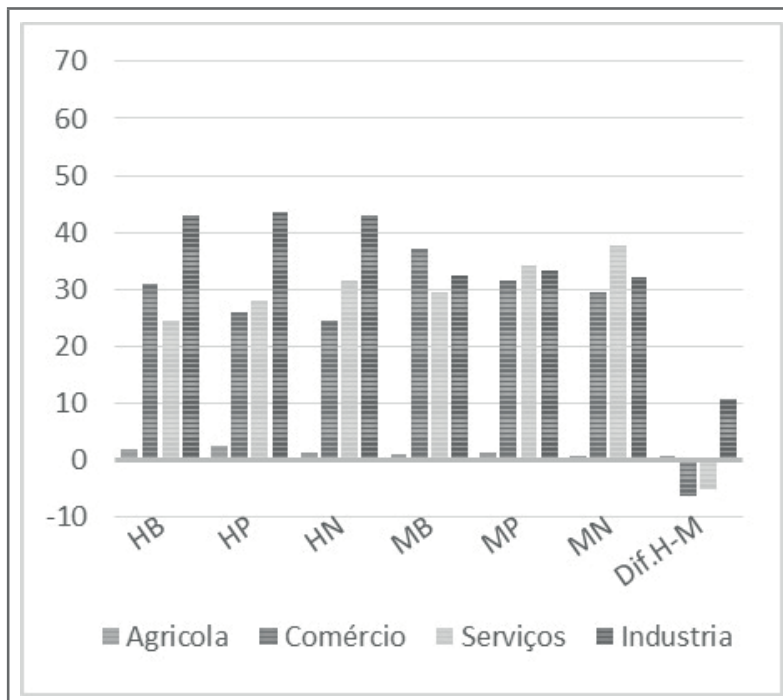

Figura 1a - Ensino médio de 18 a 24 anos

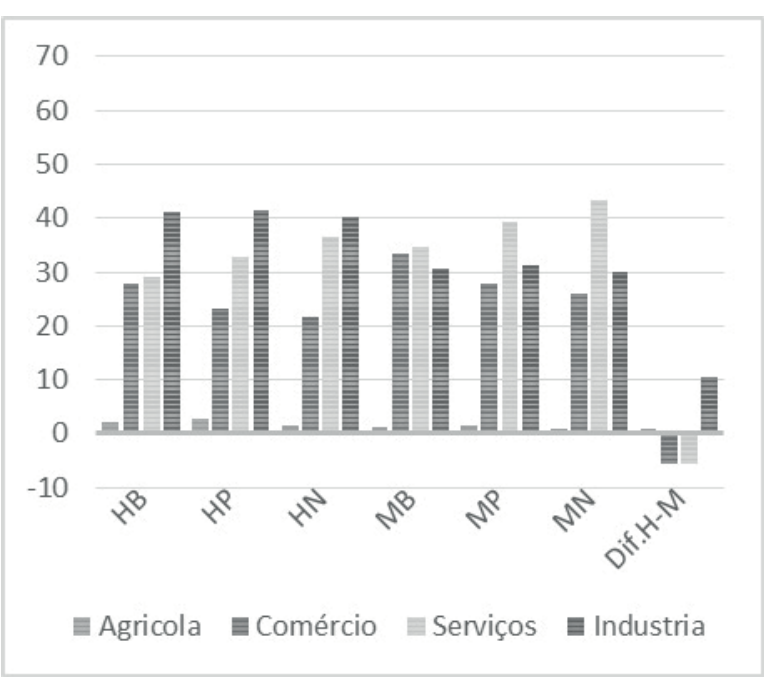

Figura 1c - Ensino médio de 25 a 29 anos

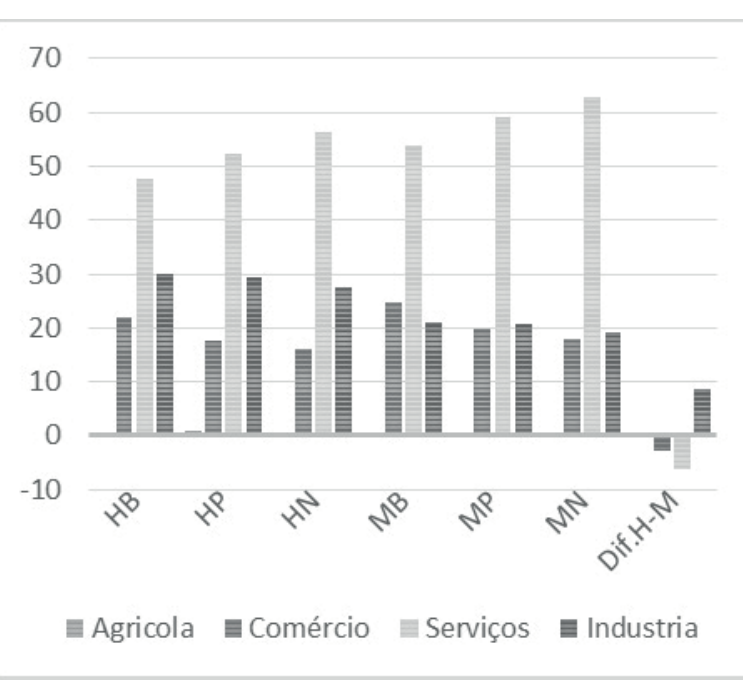

Figura 1b - Ensino superior de 18 a 24 anos

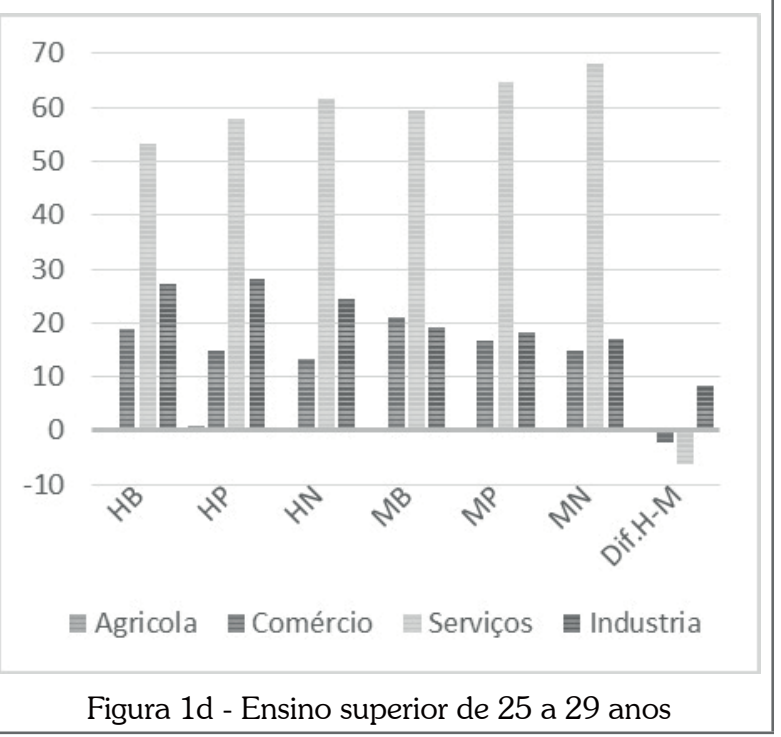

Nota 1: Ensino médio (completo e incompleto); Ensino Superior (completo e incompleto) Nota 2: Indivíduos sem experiência e média de salários

Figura 1 - Probabilidades de ingresso nos setores econômicos, em Santa Catarina

Fonte: Elaborada pelos autores deste artigo, com resultados dos efeitos marginais 
Para indivíduos com a mesma faixa etária (18 a 24 anos), porém com ensino superior (Figura 1b), o setor que apresentou maior probabilidade de inserção dessas pessoas no mercado de trabalho formal foi o setor de Serviços. No estado de Santa Catarina, homens brancos entre 18 a 24 anos com nível superior tinham mais de $47,6 \%$ de probabilidade de fazer parte do setor de Serviços. Os percentuais superaram os $56 \%$ para os homens negros, $53 \%$ para mulheres brancas e $62 \%$ para as mulheres negras.

No que tange as diferenças sexuais, os setores de Comércio e Serviços apresentam preferências para o sexo feminino. Um ponto importante a destacar é que para o nível de escolaridade do ensino superior, as diferenças de sexo no Comércio foram menores do que para o ensino médio; o setor apresentou apenas $2,87 \%$ de probabilidade a favor do sexo feminino.

No caso dos indivíduos com idade de 25 a 29 anos com ensino médio (Figura 1c), sem experiência, destacou-se o setor da Indústria como demandador de pessoas do sexo masculino. Em relação à etnia, destes trabalhadores do sexo masculino no estado de Santa Catarina, nota-se uma homogeneidade nas probabilidades de absorção.

Para a mão de obra feminina, o setor que tem maior probabilidade de fornecer um primeiro emprego com essas características foi o de Serviços. Neste setor em Santa Catarina, mulheres brancas com tais características tem uma probabilidade de conseguir um primeiro emprego de $34,6 \%$, já as mulheres pardas têm $39,4 \%$ e as mulheres negras, de $43,3 \%$.

Ao verificar as diferenças por gênero, os setores Agrícola e Indústria, dão preferência ao sexo masculino, já os setores do Comércio e Serviços, tendem a contratar mais mão de obra feminina. Na Indústria, a diferença é de $10,4 \%$ a favor do sexo masculino. Nos setores de Comércio e de Serviços a diferença é em favor do sexo feminino, $5,5 \%$ e $5,7 \%$ respectivamente para o estado.

Os indivíduos com 25 a 29 anos de idade e ensino superior (Figura1d) tem maiores oportunidades de primeira inserção no mercado de trabalho nos Serviços; os homens brancos apresentaram uma probabilidade superior a $53 \%$, os homens pardos de $54,9 \%$, e os homens negros, de mais de $61 \%$.

A probabilidade de contratação das mulheres brancas no setor de Serviços é de 55,5\%, já para as de cor parda a chance em ingressar em um primeiro emprego foi superior a $64 \%$. Para as mulheres negras, a probabilidade de fazer parte do setor foi mais de $68 \%$, comparando-se com os demais setores.

Os setores de Comércio e de Serviços também apresentaram preferências ao sexo feminino. Um ponto importante é que para indivíduos com ensino superior, as diferenças no Comércio foram menores do que para os de ensino médio. Em Santa Catarina, o Comércio apresentou uma probabilidade de $2,23 \%$ a favor da contratação de mão de obra feminina.

\section{Conclusões}

Este artigo identificou as probabilidades de inserção de pessoas no primeiro emprego formal privado no estado de Santa Catarina em 2012. O estado criou oportunidades para 90.314 indivíduos que buscaram emprego pela primeira vez, o que corresponde a $26,11 \%$ do total dos primeiros empregos gerados na região sul do Brasil.

Os setores que mais deram oportunidades para indivíduos sem experiência em Santa Catarina foram: Indústria $(34,87 \%)$, Serviços $(31,93 \%)$, Comércio $(30,37 \%)$. Indivíduos que pertenciam à faixa etária de 18 a 24 anos foram priorizados na oportunidade do primeiro emprego. As Micro e Pequenas Empresas no estado juntas correspondem a aproximadamente $48 \%$ das primeiras oportunidades de emprego formal geradas, o que aparentemente determina que esta classe de trabalhadores esteja segmentada no mercado de trabalho secundário de acordo com a teoria da segmentação.

O setor que melhor remunerou homens e mulheres sem experiência de trabalho no estado foi o de Serviços, mas também foi o que apresentou a maior diferença de salários médios em favor dos homens. O setor que apresentou menor desigualdade salarial por gênero foi o de Comércio, cuja diferença de salários entre homens e mulheres foi de $4,3 \%$.

No que tange à probabilidade de contratação nos setores, os homens e mulheres brancas que estavam na faixa etária de 18 e 24 anos e com o ensino médio, tiveram mais probabilidade de encontrar um primeiro emprego no setor da Indústria. Já as mulheres pardas e negras, tiveram a maior probabilidade de engajar 
um emprego no setor de Serviços. Considerando o nível superior de escolaridade, para ambos os sexos, o setor que forneceu a maior probabilidade de inserção foi o de Serviços.

Entre as possibilidades de inserção no mercado de trabalho de indivíduos sem experiência, com 25 a 29 anos de idade e com ensino médio, no geral, o setor que se destacou foi o da Indústria. Para o sexo feminino, o setor que apresentou maior chance de absorção foi o setor de Serviços. Nessa faixa de idade, o nível de escolaridade superior destacou-se para ambos os sexos no setor de Serviços, com as maiores probabilidades de absorção de mão de obra.

No geral, os setores de Comércio e de Serviços, deram mais chances aos trabalhadores do sexo feminino, e os setores Agrícola e da Indústria aos do sexo masculino. Quanto maior a escolaridade menores são as diferenças por gênero para os setores Agrícola, Comércio e Industria, e maiores são as preferencias pelas mulheres no setor de Serviços. O Comércio é o que apresentou a menor desigualdade de contratação entre homens e mulheres. Quando comparadas as faixas de escolaridade, quanto maior o nível de ensino menores são as diferenças de primeira contratação entre os gêneros e cor, o que pode vir a reforçar as políticas de incentivo a educação.

Este estudo, portanto, vem contribuir para o conhecimento do padrão de contratação dos trabalhadores de primeiro emprego formal no estado, bem como subsidiar as decisões de políticas públicas relativas ao primeiro emprego no estado de Santa Catarina.

\section{Agradecimentos}

Agradecemos à CAPES e ao Prof. Dr. Aricieri Devidé Junior pelos comentários aos procedimentos metodológicos.

\section{REFERÊNCIAS}

BARREIRO, J.; RUZO, E.; LOSADA, F. Modelo

Logit Multinomial: una aplicación regional al sector lacteo. Regional and Sectoral Economic Studies, [S.l.], v. 4, n. 1, 2004.
BORJAS, George. Economia do Trabalho. 5. ed. Porto Alegre: McGraw Hill Brasil, 2012.

BOUSQUAT, A.; COHN, A. A construção do mapa da juventude de São Paulo. Lua Nova, [S.I.], v. 60, p. 8196, 2003.

CACCIAMALI, M. C. S. Mercado de trabalho: abordagens duais. Revista de Administração de Empresas, [S.l.], v. 18, n. 1, p. 59-69, 1978.

\section{CARVALHO, J. D. Alguns Aspectos da Inserção} de Jovens no Mercado de Trabalho no Brasil: concepções, dados estatísticos, legislação, mecanismos de inserção e políticas públicas. São Paulo: [s.n], 2004. Disponível em: <http://www.usp.br/nce/wcp/arq/ textos/146.pdf>. Acesso em: 13 jan. 2015.

CASARI, P. Segmentação no mercado de trabalho brasileiro: diferenças entre o setor agropecuário e os setores não agropecuários, período de 2004 a 2009. 2012, p. 142f.Tese (Doutorado em Economia Aplicada) - Escola Superior de Agricultura "Luiz de Queiroz", Piracicaba, 2012.

DIEESE. Desigualdade de Raça e Gênero no Mercado de Trabalho Formal na Região Metropolitana de Campinas. Observatório do trabalho de Campinas, nov. 2009.

\section{FÁVARO, L. P. et al. Métodos quantitativos com}

STATA. Rio de Janeiro: Elsevier, 2014.

FERNANDES, R.; PICCHETTI, P. Uma análise da estrutura do desemprego e da inatividade no Brasil metropolitano. Pesquisa e Planejamento Econômico, [S.I.], v. 29, n. 1, p. 87-112, 1999.

GREENE, W. H. Econometric Analysis. 7th ed. Upper Saddle River, NJ: Prentice Hall. 2012.

IBGE. Síntese de Indicadores Sociais: uma análise das condições de vida da população brasileira.

Estudo \& Pesquisa Informações Demográficas e Socioeconômicas, Rio de Janeiro, n. 32, 2013.

KON, A. Segmentação ocupacional dos trabalhadores brasileiros segundo raça. XIV Encontro Nacional de Estudos Populacionais, ABEP, [S.1.], p. 20-24, set. 2004. 
KON, A.; LEITE, M. G.; MARQUES, R. M. Determinantes das mudanças ocupacionais no mercado de trabalho brasileiro e impactos sobre o balanço previdenciário.

Ciclo de debates em economia industrial, trabalho $e$ tecnologia, São Paulo, PUC-SP, 2011.

LIMA, R. Mercado de trabalho: o capital humano e a teoria da segmentação. Pesquisa e Planejamento

Econômico, [S.l.], v. 10, n. 1, p. 217-272, 1980.

LONG, J. S.; FREESE, J. Regression models for categorical dependent variables using Stata. College Station, 2001.

MENDONÇA, T. M. et al. Determinantes da Inserção de Mulheres Jovens no Mercado de Trabalho

Nordestino. Revista de Economia do Nordeste, [S.l.], v. 43, n. 4, p. 161-174, 2012.

OLIVEIRA, P. R.; SCORZAFAVE, L. G.; PAZELLO, E. T. Desemprego e inatividade nas metrópoles brasileiras: as diferenças entre homens e mulheres. Nova Economia, [S.l.], v. 19, n. 2, p. 291-324, 2009.

POCHMANN, Márcio. (Org.). Outra cidade é possível: alternativas de inclusão social em São Paulo. São Paulo: Cortez, 2003.
REMY, M. A. P. A.; VAZ, D. V. Determinantes que impactam na probabilidade de o jovem não participar do mercado de trabalho e simultaneamente não estudar. In: XIX ENCONTRO NACIONAL DE ESTUDOS POPULACIONAIS, 2012, São Pedro. Anais... São Pedro/ SP, 2014. p. 8-31.

SEBRAE. Anuário do trabalho na micro e pequena empresa: 2013. 6. ed. Brasília, DF: DIEESE, 2013.

SILVA, N. D. V.; KASSOUF, A. L. A exclusão social dos jovens no mercado de trabalho brasileiro. Revista Brasileira de Estudos de População, [S.l.], v. 19, n. 2, p. 99-115, jul./dez. 2002.

TOMÁS M. C. O ingresso dos jovens no mercado de trabalho: uma análise das regióes metropolitanas brasileiras nas últimas décadas. 2007, p. $168 f$. Dissertação (Mestrado em Demografia) - Universidade Federal de Minas Gerais, 2007.

VIETORISZ, T.; HARRISON, B. Labor Market Segmentation: Positive feedback and divergent development. American Economic Review, [S.l.], v. 63, n. 2, p. 366-376, May, 1973. 


\section{Apêndice A - Resultados da regressão logit Multinomial, Santa Catarina, 2012}

A1 - Resultados da regressão Logit Multinomial, Santa Catarina, 2012

\begin{tabular}{|c|c|c|c|c|c|c|}
\hline \multirow{2}{*}{ SETOR/VARIÁveIS } & \multicolumn{2}{|c|}{ Agricultura } & \multicolumn{2}{|c|}{ ComÉrcio } & \multicolumn{2}{|c|}{ INDÚSTRIA } \\
\hline & RRR & STD. ERR. & RRR & STD. ERR. & RRR & STD. ERR. \\
\hline Preta & $0,590 *$ & 0,034 & $0,616^{*}$ & 0,015 & 0,778* & 0,015 \\
\hline Parda & $1,225^{*}$ & 0,041 & 0,730* & 0,013 & $\mathbf{0 , 8 8 8 ^ { * }}$ & 0,013 \\
\hline Homem & $2,083^{*}$ & 0,040 & $0,996^{\mathrm{ns}}$ & 0,007 & $1,598^{*}$ & 0,011 \\
\hline Experiência & $0,980 *$ & 0,003 & $0,996^{*}$ & 0,002 & $0,997 * *$ & 0,001 \\
\hline Experiência2 & $1,000^{\mathrm{ns}}$ & 0,000 & $1,000^{\text {ns }}$ & 0,000 & $1,000 * *$ & 0,000 \\
\hline Analfabeto & $9,065^{*}$ & 0,847 & $0,522^{*}$ & 0,045 & $1,923^{*}$ & 0,110 \\
\hline Primário & $7,632 *$ & 0,221 & $0,382 *$ & 0,009 & $1,407 *$ & 0,022 \\
\hline Fundamental & $4,051^{*}$ & 0,087 & $0,758 *$ & 0,008 & 1,591* & 0,014 \\
\hline Superior & $0,168^{*}$ & 0,011 & $0,366^{*}$ & 0,004 & $0,359 *$ & 0,004 \\
\hline Idade15_A_17 & $1,187^{*}$ & 0,053 & $1,581 *$ & 0,027 & $0,908 *$ & 0,016 \\
\hline Idade25_A_29 & $0,980^{\text {ns }}$ & 0,030 & $0,766^{*}$ & 0,009 & $\mathbf{0 , 8 1 2 *}$ & 0,009 \\
\hline Idade30_A_39 & $1,105^{*}$ & 0,035 & $0,676 *$ & 0,010 & $0,756^{*}$ & 0,010 \\
\hline Idade40_A_49 & $1,283^{*}$ & 0,054 & $0,648^{*}$ & 0,014 & $0,752 *$ & 0,014 \\
\hline Idade50_A_64 & $1,280 *$ & 0,087 & $0,624 *$ & 0,023 & $0,656^{*}$ & 0,020 \\
\hline _cons & $\mathbf{0 , 0 3 4} *$ & 0,001 & $1,264^{*}$ & 0,012 & $1,096^{*}$ & 0,009 \\
\hline
\end{tabular}

Nota1: Categoria base: Serviços; * significância de 1\%;** significância de 5\%; *** significância de 10 \%; ns: não significativo. Nota 2: Foram feitos teste de Hausman ${ }^{1} e$ Teste Wald ${ }^{2}$.

\footnotetext{
${ }^{1}$ Para o teste de Hausman, ver Long e Free (2001). Segundo Long e Free (2001), o modelo Logit Multinomial se baseia no pressuposto de independência de alternativas irrelevantes - Independence of Irrelevant Alternatives, (IIA). Para as categorias omitidas, os resultados dos qui-quadro (Chi2) foram negativos, o que segundo Hausman e McFedden (1984) apud Long e Free (2001), são evidências de que a hipótese de que a IIA não foi violada.

${ }^{2}$ Para o teste Wald, - "Combinação entre as categorias", ver Long e Free (2001. Os valores de P>Chi2 foram significativos a 1\% de significância, o que indica que se rejeita a hipótese nula, portanto, não é aconselhável que se combine as categorias.
} 